\title{
The Hippo Signaling Pathway in Pancreatic Cancer
}

\author{
DANIEL ANSARI ${ }^{1}$, HENRIK OHLSSON $^{1}$, CARL ALTHINI $^{1}$, MONIKA BAUDEN $^{1}$, \\ QIMIN ZHOU ${ }^{1,2}$, DINGYUAN HU ${ }^{1,3}$ and ROLAND ANDERSSON ${ }^{1}$ \\ ${ }^{I}$ Department of Surgery, Clinical Sciences Lund, Lund University and Skåne University Hospital, Lund, Sweden; \\ ${ }^{2}$ The Eye Hospital, School of Ophthalmology and Optometry, \\ Wenzhou Medical University, Zhejiang, P.R. China; \\ ${ }^{3}$ Department of Gastroenterology, The Second Affiliated Hospital and \\ Yuying Children's Hospital of Wenzhou Medical University, Wenzhou, P.R. China
}

\begin{abstract}
Hippo signaling is a key regulator of organ size, tissue hemostasis and regeneration. Dysregulation of the Hippo pathway has been recognized in a variety of human cancers, including pancreatic cancer. YES-associated protein $(Y A P)$ and transcriptional coactivator with PDZ-binding motif (TAZ) are the two major downstream effectors of the Hippo pathway. YAP and TAZ have been found to promote pancreatic tumor development and progression, even in the absence of mutant Kirsten RAS (KRAS). Pancreatic cancer is associated with an abundant stromal reaction leading to tumor growth and immune escape. It has been found that YAP and TAZ modulate behavior of pancreatic stellate cells and recruitment of tumor-associated macrophages and myeloid-derived suppressor cells. Moreover, YAP and TAZ are associated with chemoresistance and poor prognosis in pancreatic cancer. This review dissects the role of Hippo signaling in pancreatic cancer, focusing on molecular mechanisms and prospects for future intervention.
\end{abstract}

Pancreatic cancer is the third most common cause of cancerrelated death (1). Despite advances in surgery and chemotherapy, the overall prognosis has remained virtually unchanged for many decades (2). The median survival of patients with pancreatic cancer, overall, is about 6 months and

This article is freely accessible online.

Correspondence to: Professor Roland Andersson, MD, Ph.D., Department of Surgery, Clinical Sciences Lund, Lund University and Skåne University Hospital, 22185 Lund, Sweden. E-mail: roland.andersson@med.lu.se

Key Words: Pancreatic Cancer, Hippo pathway, YAP, TAZ, tumor progression, EMT, stroma, immunomodulation, chemoresistance, treatment, review. the 5-year survival rate is below 5\% (3). Most patients are diagnosed with disease at late stages due to lack of early symptoms and accurate diagnostic markers. Fewer than $20 \%$ of patients have up-front resectable disease. However, even after radical resection, most patients develop recurrence and die of their disease. Pancreatic cancer is a heterogenous disease harboring multiple genomic, proteomic and epigenetic alterations related to alterations in several core signaling pathways (4). The complex interplay between cancer cells and the surrounding stroma also represents a challenge to development of treatment (5). These statistics highlight the need for new approaches to pancreatic cancer diagnosis and therapy.

The Hippo signaling pathway was first discovered from studies in Drosophila melanogaster (6). Under physiological conditions, Hippo signaling governs normal organ development and tissue regeneration (7). Deregulation of Hippo signaling has been recognized in a variety of human malignancies, including pancreatic cancer $(8,9)$. Deregulation of the Hippo pathway has been related to tumor cell behavior, as well as prognosis in patients with cancer (9). The Hippo pathway has become extensively studied in pancreatic cancer and results of these studies have corroborated the biological importance of this pathway.

In this review, we summarize the mechanisms by which Hippo signaling affects pancreatic cell growth, invasion and metastatic behavior. We further discuss how Hippo signaling interacts with the stroma and host immune response. Finally, we review the role of Hippo signaling in treatment resistance and clinical outcome.

\section{The Hippo Signaling Pathway}

Hippo signaling is an evolutionarily conserved network that plays a key role in regulating cell proliferation, organ growth and regeneration (Figure 1). Its core consists of the serine/threonine kinases mammalian sterile 20-like kinase 1 
and 2 (MST1 and MST2, HPO in Drosophila) and large tumor suppressor 1 and 2 (LATS1 and LATS2). MST1 and -2 work with Salvador homolog 1 (SAV1) to phosphorylate and activate LATS1 and -2 kinases. Subsequently, LATS1 and -2 kinases combine with the adaptor, MOB kinase activator 1 (MOB1), and result in the phosphorylation of the Hippo transducers YES-associated protein (YAP) and transcriptional co-activator with PDZ-binding motif (TAZ). By phosphorylation, YAP and TAZ are prevented from nuclear accumulation and interaction with transcription factors such as TEA transcriptional factor (TEAD)-1, TEAD2, TEAD3 and TEAD4. If the regulatory module is inactivated, or in the presence of stimuli that activate YAP/TAZ independently from the Hippo kinases, the Hippo transducers YAP/TAZ accumulate in the nucleus and interact with transcriptional factors, thereby mediating transcription of target genes such as connective tissue growth factor $(C T G F)$, cysteine-rich angiogenic inducer 61 (CYR61), ankyrin repeat domain 1 (ANKRD1), baculoviral inhibitor of apoptosis repeatcontaining 5 (BIRC5) and AXL receptor tyrosine kinase (AXL) (7). Multiple inputs control Hippo signaling, spanning from mechanical cues instructed by the cellular microenvironment (mechano-transduction) to soluble factors and metabolic pathways $(10,11)$. The Hippo pathway is involved in extensive crosstalk with other signaling pathways such as transforming growth factor-beta, Wnt, Sonic hedgehog and Notch (12).

\section{YAP Expression in Pancreatic Cancer}

Several studies have indicated that YAP is overexpressed in tumor samples from patients with pancreatic cancer (13-17). Activating mutations in KRAS are among the most frequent events in pancreatic cancer. YAP has been identified as a critical partner to mutant KRAS in driving pancreatic carcinogenesis in experimental models. YAP reportedly acts as a transcriptional switch downstream of KRAS, promoting expression of genes that promote neoplastic proliferation, stromal response and the progression to invasive carcinoma (18). Furthermore, it has been found that deletion of the tumor suppressor F-box and WD repeat domain containing 7 $(F B X W 7)$ accelerates KRAS-driven pancreatic tumorigenesis via YAP accumulation (19). At the same time, it has been demonstrated that tumor cells can survive without KRAS signaling, with bypass mechanisms involving YAP (13).

\section{Tumor Growth}

There is accumulating evidence that YAP and TAZ promote proliferation and growth of pancreatic cancer cells. Treatment of pancreatic cancer cells with YAP-targeting small interfering RNA oligonucleotides significantly reduced tumor growth (16). It has been reported that eukaryotic translation initiation factor 5A (eIF5A)-pseudopodium enriched atypical kinase 1 (PEAK1) signaling regulates YAP and TAZ expression and pancreatic cancer cell growth. Disrupting eIF5A-PEAK1 signaling in pancreatic cancer cells inhibited protein expression to YAP and TAZ, reducing expression of stem cellassociated transcription factors and tumor sphere growth (20).

\section{Epithelial-Mesenchymal Transition (EMT), Invasion and Metastasis}

EMT is a developmental regulatory program defined by the phenotypical transition from an epithelial to a mesenchymal cell state. EMT is an essential step for metastasis and confers resistance to therapy. Active YAP promotes pancreatic cancer cell motility, invasion and tumorigenesis in a mitotic phosphorylation-dependent manner and contributes to EMT in pancreatic cancer cells by several mechanisms, including hyperactivation of AKT signaling $(14,15,21,22)$.

\section{Stroma}

Pancreatic cancer is characterized by a dense stroma that can promote tumor growth. The stroma contains cancer-associated fibroblasts (CAFs), immune cells, endothelial cells and extracellular matrix. Pancreatic stellate cells are resident mesenchymal cells of the pancreas that represent the major source of CAFs. It has been found that YAP and TAZ are expressed at high levels in activated pancreatic stellate cells in pancreatic cancer, as well as in chronic pancreatitis (23). Transglutaminase 2 secreted by pancreatic cancer cells modulates the stroma by cross-linking collagen, which, in turn, activates CAFs and pancreatic stellate cells and stimulates their proliferation. The stiff fibrotic stromal reaction confers mechanical signals to cancer cells, leading to activation of the YAP and TAZ transcription factors and tumor progression (24).

\section{Immunomodulation}

Pancreatic cancer is characterized by a profound inflammatory reaction and immunosuppressive state (25). Pancreatic tumors are associated with immune dysfunction, partly mediated by the recruitment of immunosuppressive cells, such as tumorassociated macrophages and myeloid-derived suppressor cells (26-28). These cells are recruited to the tumor microenvironment and can inhibit T-cell activity. YAP has been identified as a critical regulator of the immunosuppressive microenvironment in pancreatic cancer. YAP inactivation prevented recruitment of myeloid-derived suppressor cells while in turn supporting infiltration of antigen presenting macrophages and T-cell activation, thereby promoting apoptosis of tumor cells (29).

\section{Chemoresistance}

Gemcitabine has been the standard chemotherapeutic agent in pancreatic cancer since 1997 (30). However, pancreatic cancer 


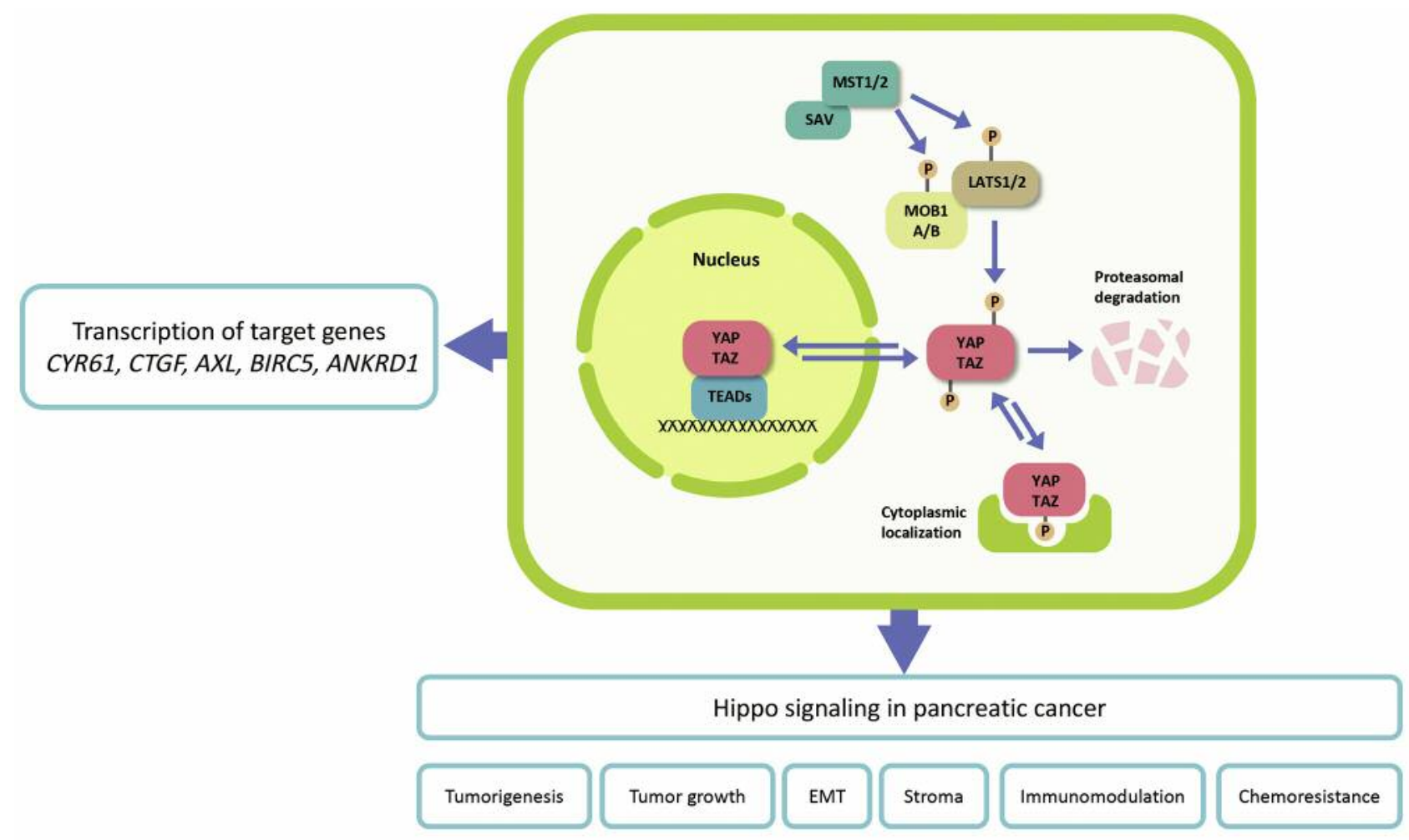

Figure 1. The Hippo signaling pathway. Mammalian sterile 20-like kinase 1 and 2 (MST1/2) work with scaffolding proteins Salvador homolog (SAV) to phosphorylate and activate large tumor suppressor 1 and 2 (LATS1/2) kinases. Subsequently, LATS1/2 kinases combine with the adaptor, MOB kinase activator $1 A$ and $1 B(M O B 1 A / B)$, and result in the phosphorylation of the Hippo transducers YES-associated protein (YAP) and transcriptional co-activator with PDZ-binding motif (TAZ). By phosphorylation, YAP and TAZ are retained in the cytoplasm or degraded and thereby prevented from nuclear accumulation and interaction with transcription factors such as TEA transcriptional factor (TEAD)-1, TEAD2, TEAD3 and TEAD4. Aberrant Hippo signaling in pancreatic cancer is related to tumorigenesis, tumor growth, epithelial-mesenchymal transition (EMT), stroma, immunomodulation and chemoresistance. ANKRD1: Ankyrin repeat domain 1; AXL: AXL receptor tyrosine kinase; BIRC5: baculoviral inhibitor of apoptosis repeat-containing 5; CTGF: connective tissue growth factor; CYR61: cysteine-rich angiogenic inducer 61.

is characterized by a high degree of chemoresistance. Several mechanisms of YAP-induced chemoresistance have been proposed. One mechanism suggests that YAP overexpression induces EMT in pancreatic cancer cells by activating the AKT cascade, which can cause resistance to gemcitabine (21). Another mechanism involves microRNAs. It has been suggested that microRNA 181c is overexpressed in pancreatic cancer samples and correlated with poor prognosis. MicroRNA 181c directly repressed MST1, LATS2, MOB1 and $S A V 1$, leading to YAP and TAZ activation, and gemcitabine resistance in vitro and in vivo (31).

\section{Patient Survival}

Despite extensive research, no single prognostic molecular marker is used in the clinical management of pancreatic cancer. The clinical significance of YAP as a prognostic marker has been investigated in several studies. It has been found that nuclear overexpression of YAP is an independent prognostic marker for poor survival and is associated with liver metastasis (17). Furthermore, using public messenger RNA expression data, YAP was confirmed to be correlated with poor survival (32). The 5-year survival rate was $0 \%$ in patients with high YAP mRNA expression compared to $32 \%$ in those with low expression. Furthermore, multiple YAP/TEAD-regulated genes were associated with poor prognosis, such as transforming growth factor alpha $(T G F \alpha)$, heparin binding EGF like growth factor $(H B E G F)$, integrin subunit alpha 2 (ITGA2), P2Y2 receptor $(P 2 Y 2 R), \mathrm{G}$ proteincoupled receptor 87 (GPR87) and mucin 1 (MUC1). On the other hand, YAP-inhibitory pathways were associated favorable prognosis, such as STE20-related kinase adaptor/liver kinase B1 (STRAD/LKB-1), protein kinase A/large tumor suppressor (PKA/LATS), and tuberous sclerosis complex/mammalian target of rapamycin complex 1 (TSC/mTORC1). 


\section{Therapeutic Targeting of Hippo Signaling}

Considering the role of YAP and TAZ in pancreatic tumor biology and clinical outcome, it is reasonable to develop drugs that target YAP and TAZ activity. Of the evaluated treatments in pancreatic cancer to date, only verteporfin $(33,34)$ has a direct effect upon Hippo signaling. Erlotinib (35), BIS 1 (35) and LY3009120 (33) indirectly affect YAP and TAZ signaling. The mechanism of action is not fully clarified for several natural substances, such as curcumin (36), resveratrol (37), Stiehopus japonieus acidic mucopolysaccharide (38) and pseudolaric acid B (39).

\section{Conclusion}

The Hippo pathway is an evolutionary conserved signaling pathway, involved in organ size control and tissue homeostasis. YAP and TAZ are key downstream regulators in the Hippo pathway that are found to be up-regulated in pancreatic cancer. Aberrant transcriptional activity of YAP and TAZ has a crucial role in pancreatic tumor cell biology, including growth, EMT, microenvironmental signaling and drug resistance. YAP and TAZ are prognostic factors in pancreatic cancer. In the future, direct or indirect pharmacological modulation of the Hippo pathway or YAP and TAZ targeting genes may become promising approaches to treat pancreatic cancer.

\section{References}

1 Siegel RL, Miller KD and Jemal A: Cancer statistics, 2018. CA Cancer J Clin 68(1): 7-30, 2018.

2 Ansari D, Tingstedt B, Andersson B, Holmquist F, Sturesson C, Williamsson C, Sasor A, Borg D, Bauden M and Andersson R: Pancreatic cancer: Yesterday, today and tomorrow. Future Oncol 12(16): 1929-1946, 2016.

3 Carrato A, Falcone A, Ducreux M, Valle JW, Parnaby A, Djazouli K, Alnwick-Allu K, Hutchings A, Palaska C and Parthenaki I: A systematic review of the burden of pancreatic cancer in europe: Real-world impact on survival, quality of life and costs. J Gastrointest Cancer 46(3): 201-211, 2015.

4 Torres C and Grippo PJ: Pancreatic cancer subtypes: A roadmap for precision medicine. Ann Med 50(4): 277-287, 2018.

5 Ansari D, Carvajo M, Bauden M and Andersson R: Pancreatic cancer stroma: Controversies and current insights. Scand J Gastroenterol 52(6-7): 641-646, 2017.

6 Harvey KF, Pfleger CM and Hariharan IK: The Drosophila mst ortholog, hippo, restricts growth and cell proliferation and promotes apoptosis. Cell 114(4): 457-467, 2003.

7 Maugeri-Sacca M and De Maria R: The Hippo pathway in normal development and cancer. Pharmacol Ther 186: 60-72, 2018.

8 Yu FX, Zhao B and Guan KL: Hippo pathway in organ size control, tissue homeostasis, and cancer. Cell 163(4): 811-828, 2015.

9 Poma AM, Torregrossa L, Bruno R, Basolo F and Fontanini G: Hippo pathway affects survival of cancer patients: Extensive analysis of TCGA data and review of literature. Sci Rep 8(1): 10623, 2018.

10 Piccolo S, Dupont S and Cordenonsi M: The biology of YAP/TAZ: Hippo signaling and beyond. Physiol Rev 94(4): 1287-1312, 2014.

11 Dupont S, Morsut L, Aragona M, Enzo E, Giulitti S, Cordenonsi M, Zanconato F, Le Digabel J, Forcato M, Bicciato S, Elvassore $\mathrm{N}$ and Piccolo S: Role of YAP/TAZ in mechanotransduction. Nature 474(7350): 179-183, 2011.

12 Yu FX, Meng Z, Plouffe SW and Guan KL: Hippo pathway regulation of gastrointestinal tissues. Annu Rev Physiol 77: 201227, 2015.

13 Kapoor A, Yao W, Ying H, Hua S, Liewen A, Wang Q, Zhong Y, Wu CJ, Sadanandam A, Hu B, Chang Q, Chu GC, Al-Khalil R, Jiang S, Xia H, Fletcher-Sananikone E, Lim C, Horwitz GI, Viale A, Pettazzoni P, Sanchez N, Wang H, Protopopov A, Zhang J, Heffernan T, Johnson RL, Chin L, Wang YA, Draetta G and DePinho RA: Yap1 activation enables bypass of oncogenic kras addiction in pancreatic cancer. Cell 158(1): 185-197, 2014.

14 Xie D, Cui J, Xia T, Jia Z, Wang L, Wei W, Zhu A, Gao Y, Xie $\mathrm{K}$ and Quan M: Hippo transducer TAZ promotes epithelialmesenchymal transition and supports pancreatic cancer progression. Oncotarget 6(34): 35949-35963, 2015.

15 Yang S, Zhang L, Purohit V, Shukla SK, Chen X, Yu F, Fu K, Chen Y, Solheim J, Singh PK, Song W and Dong J: Active YAP promotes pancreatic cancer cell motility, invasion and tumorigenesis in a mitotic phosphorylation-dependent manner through LPAR3. Oncotarget 6(34): 36019-36031, 2015.

16 Diep CH, Zucker KM, Hostetter G, Watanabe A, Hu C, Munoz RM, Von Hoff DD and Han H: Down-regulation of YESassociated protein 1 expression reduces cell proliferation and clonogenicity of pancreatic cancer cells. PLoS One 7(3): e32783, 2012.

17 Salcedo Allende MT, Zeron-Medina J, Hernandez J, Macarulla T, Balsells J, Merino X, Allende H, Tabernero J and Ramon YCS: Overexpression of YES-associated protein 1, an independent prognostic marker in patients with pancreatic ductal adenocarcinoma, correlated with liver metastasis and poor prognosis. Pancreas 46(7): 913-920, 2017.

18 Zhang W, Nandakumar N, Shi Y, Manzano M, Smith A, Graham G, Gupta S, Vietsch EE, Laughlin SZ, Wadhwa M, Chetram M, Joshi M, Wang F, Kallakury B, Toretsky J, Wellstein A and Yi C: Downstream of mutant KRAS, the transcription regulator YAP is essential for neoplastic progression to pancreatic ductal adenocarcinoma. Sci Signal 7(324): ra42, 2014.

19 Zhang Q, Zhang Y, Parsels JD, Lohse I, Lawrence TS, Pasca di Magliano M, Sun Y and Morgan MA: FBXW7 deletion accelerates KRAS(G12D)-driven pancreatic tumorigenesis via YAP accumulation. Neoplasia 18(11): 666-673, 2016.

20 Strnadel J, Choi S, Fujimura K, Wang H, Zhang W, Wyse M, Wright T, Gross E, Peinado C, Park HW, Bui J, Kelber J, Bouvet M, Guan KL and Klemke RL: EIF5A-PEAK1 signaling regulates YAP1/TAZ protein expression and pancreatic cancer cell growth. Cancer Res 77(8): 1997-2007, 2017.

21 Yuan Y, Li D, Li H, Wang L, Tian G and Dong Y: YAP overexpression promotes the epithelia--mesenchymal transition and chemoresistance in pancreatic cancer cells. Mol Med Rep 13(1): 237-242, 2016.

22 Wei H, Xu Z, Liu F, Wang F, Wang X, Sun X and Li J: Hypoxia induces oncogene YES-associated protein 1 nuclear translocation 
to promote pancreatic ductal adenocarcinoma invasion via epithelial-mesenchymal transition. Tumour Biol 39(5): 10104283 17691684, 2017.

23 Morvaridi S, Dhall D, Greene MI, Pandol SJ and Wang Q: Role of YAP and TAZ in pancreatic ductal adenocarcinoma and in stellate cells associated with cancer and chronic pancreatitis. Sci Rep 5: 16759, 2015.

24 Lee J, Condello S, Yakubov B, Emerson R, Caperell-Grant A, Hitomi K, Xie J and Matei D: Tissue transglutaminase mediated tumor-stroma interaction promotes pancreatic cancer progression. Clin Cancer Res 21(19): 4482-4493, 2015.

25 von Bernstorff W, Voss M, Freichel S, Schmid A, Vogel I, Johnk C, Henne-Bruns D, Kremer B and Kalthoff H: Systemic and local immunosuppression in pancreatic cancer patients. Clin Cancer Res 7(3 Suppl): 925s-932s, 2001.

26 Mace TA, Ameen Z, Collins A, Wojcik S, Mair M, Young GS, Fuchs JR, Eubank TD, Frankel WL, Bekaii-Saab T, Bloomston $\mathrm{M}$ and Lesinski GB: Pancreatic cancer-associated stellate cells promote differentiation of myeloid-derived suppressor cells in a STAT3-dependent manner. Cancer Res 73(10): 3007-3018, 2013

27 Stromnes IM, Brockenbrough JS, Izeradjene K, Carlson MA, Cuevas C, Simmons RM, Greenberg PD and Hingorani SR: Targeted depletion of an MDSC subset unmasks pancreatic ductal adenocarcinoma to adaptive immunity. Gut 63(11): 17691781, 2014.

28 Mielgo A and Schmid MC: Impact of tumour-associated macrophages in pancreatic cancer. BMB Rep 46(3): 131-138, 2013.

29 Murakami S, Shahbazian D, Surana R, Zhang W, Chen H, Graham GT, White SM, Weiner LM and Yi C: YES-associated protein mediates immune reprogramming in pancreatic ductal adenocarcinoma. Oncogene 36(9): 1232-1244, 2017.

30 Burris HA, 3rd, Moore MJ, Andersen J, Green MR, Rothenberg ML, Modiano MR, Cripps MC, Portenoy RK, Storniolo AM, Tarassoff P, Nelson R, Dorr FA, Stephens CD and Von Hoff DD: Improvements in survival and clinical benefit with gemcitabine as first-line therapy for patients with advanced pancreas cancer: A randomized trial. J Clin Oncol 15(6): 2403-2413, 1997.

31 Chen M, Wang M, Xu S, Guo X and Jiang J: Upregulation of mir-181c contributes to chemoresistance in pancreatic cancer by inactivating the Hippo signaling pathway. Oncotarget 6(42): 44466-44479, 2015.
32 Rozengurt E, Sinnett-Smith J and Eibl G: YES-associated protein (YAP) in pancreatic cancer: At the epicenter of a targetable signaling network associated with patient survival. Signal Transduct Target Ther 3: 11, 2018.

33 Zhao X, Wang X, Fang L, Lan C, Zheng X, Wang Y, Zhang Y, Han X, Liu S, Cheng K, Zhao Y, Shi J, Guo J, Hao J, Ren H and Nie G: A combinatorial strategy using YAP and pan-RAF inhibitors for treating KRAS-mutant pancreatic cancer. Cancer Lett 402: 61-70, 2017.

34 Wei H, Wang F, Wang Y, Li T, Xiu P, Zhong J, Sun X and Li J: Verteporfin suppresses cell survival, angiogenesis and vasculogenic mimicry of pancreatic ductal adenocarcinoma via disrupting the YAP-TEAD complex. Cancer Sci 108(3): 478-487, 2017.

35 Thongon N, Castiglioni I, Zucal C, Latorre E, D'Agostino V, Bauer I, Pancher M, Ballestrero A, Feldmann G, Nencioni A and Provenzani A: The GSK3beta inhibitor BIS I reverts YAPdependent EMT signature in PDAC cell lines by decreasing SMADs expression level. Oncotarget 7(18): 26551-26566, 2016.

36 Zhou X, Su J, Feng S, Wang L, Yin X, Yan J and Wang Z: Antitumor activity of curcumin is involved in down-regulation of YAP/TAZ expression in pancreatic cancer cells. Oncotarget 7(48): 79076-79088, 2016.

37 Jiang Z, Chen X, Chen K, Sun L, Gao L, Zhou C, Lei M, Duan W, Wang Z, Ma Q and Ma J: YAP inhibition by resveratrol via activation of AMPK enhances the sensitivity of pancreatic cancer cells to gemcitabine. Nutrients 8(10): 546, 2016.

38 Li X, Liu Y, Zhang C, Niu Q, Wang H, Che C, Xie M, Zhou B, Xu Y, Zhang Q, Wu J and Tian Z: Stiehopus japonieus acidic mucopolysaccharide inhibits the proliferation of pancreatic cancer SW1990 cells through Hippo-YAP pathway. Oncotarget 8(10): 16356-16366, 2017.

39 Li X, Zhao X, Song W, Tian Z, Yang L, Niu Q, Zhang Q, Xie M, Zhou B, Xu Y, Wu J and Zhang C: Pseudolaric acid B inhibits proliferation, invasion and epithelial-to-mesenchymal transition in human pancreatic cancer cell. Yonsei Med J 59(1): 20-27, 2018.
Received December 10, 2018

Revised December 13, 2018

Accepted December 14, 2018 